\title{
ESTERILIZACION FEMENINA AMBULATORIA POR LAPAROSCOPIA BAJO ANESTESIA LOCAL
}

Los seminarios de adiestramiento y el trabajo en Laparoscopia, se han realizado en parte con el auspicio financiero de la Fundación Pathfinder de Boston, a quien agradecemos el aporte.

\author{
Dr. Guillermo López-Escobar, M.D.* \\ Dr. Eduardo Cáceres Alvarez, M.D.** \\ Dr. Luis Armando Muñoz, M.D.***
}

\section{Introducción}

La esterilización femenina como método quirúrgico para controlar la fecundidad tiene orígenes antiguos. Posiblemente fue el Dr. James Blundell de Londres, quien primero introdujo la ligadura de trompas en 1823. (Citado por Pai - 13). Sin embargo, ya en tiempos de Hipócrates se dice que la esterilización femenina era aconsejada para eliminar la perpetuación de enfermedades mentales (2) y Noonan anota que el Talmud menciona las mujeres que han sido "divididas o partidas" en un contexto que no explica la operación, pero sugiere que la esterilización en alguna forma era conocida (12).

La esterilización femenina por bloqueo o sección de las trompas de Falopio para impedir que el óvulo liberado por los ovarios pueda ser fecundado por los espermatozoides depositados en la vagina, era hasta hace poco tiempo una intervención que requería varios días de hospitalización, anestesia general, y exigía semanas de recuperación. Aún así, era solicitada como única solución por aquellas mujeres que no deseaban una anticoncepción temporal sino definitiva.

\section{La esterilización por laparoscopia}

Ha sido sólo en la última década que han surgido nuevas técnicas que prometen hacer de la esterilización femenina una operación de corta duración, practicable sin necesidad de internar a la paciente en el hospital. Actualmente la esterilización por Laparoscopia representa un procedimiento ambulatorio que puede llevarse a cabo en clínicas y hospitales fuera del área quirúrgica, bajo anestesia local y que termina con la colocación en la piel de una "curita" proporcionando protección casi completa contra futuros embarazos.

La Laparoscopia es por definición, un examen endoscópico del interior de la cavidad peritoneal por medio

\footnotetext{
* Presidente Corporación Centro Regional de Población, Bogotá.

** Director Servicio de Laparoscopia I.M.I. Bo-

*** Miembro Servicio de Laparoscopia I.M.I. Bogotá.
} gotá. 
de un tubo o laparoscopio introducido a través de la pared anterior del abdomen. También es conocida como peritoneoscopia o celioscopia. Se originó a comienzos del siglo, cuando algunos médicos investigadores usaron el cistoscopio de Nitze con velas para iluminar, a fin de estudiar la cavidad abdominal en perros. (Kelling, citado por Steptoe - 18) por vez primera utilizó un instrumento óptico para ejecutar exámenes celioscópicos en perros en 1901. Jacobeaus (citado por Steptoe - 10), en 1910 fue el primero en describir lo que él Ilamó "Laparoscopia", en seres humanos, utilizando un cistoscopio y fue el primero en observar y fotografiar el canal genital femenino por este medio, alrededor del año de 1912.

El Laparoscopio, tubo largo y delgado de acero inoxidable, utiliza en los aparatos modernos haces o fibras ópticas para transmitir la luz fría originada en el exterior del aparto y dirigirla hacia el interior de la cavidad abdominal.

La esterilización laparoscópica es una combinación de dos procedimientos -laparoscopia y esterilización tubárica-. Originalmente utilizada como técnica de diagnóstico la Laparoscopia fue propuesta como método de esterilización tubárica por A. T. Anderson en 1937 (1), y luego descrita por Power y Barnes de la Universidad de Michigan en 1941 (17). Palmer al informar en Paris acerca de 250 casos en 1947 (14) recomendó una cánula de permeabilidad uterina para mejor visión y manipulación. A principios de la década de 1961 el desarrollo de la transmisión óptica por medio de haces o cilindros fibrosos (la fibroscopia) abrió nuevos horizontes para la Laparoscopia. Palmer en Francia (15), Fragenheim en Alemania (8), y Steptoe en Inglaterra (18), informaron con entusiasmo acerca de las posibilidades en desarrollo. A principios de la década actual, la búsqueda mundial para encontrar mejores técnicas de anticoncepción, ha estimulado el incremento en las investigaciones, la educación, la instrumentación y el interés público en la aceptación de la esterilización laparoscópica. De las técnicas iniciales por doble incisión, se ha evolucionado a las técnicas de una sola incisión, por lo general a nivel umbilical y, hacia el uso de anestesia local simplificando la intervención.

Un buen número de cirujanos ha presentado informes acerca de las esterilizaciones laparoscópicas llevadas a cabo en los últimos años en cientos de pacientes. Para citar sólo algunos de los más importantes: la serie de 760 casos reportada en 1970 por Jordan y colaboradores (10) en Inglaterra 810 casos informados en 1972 por Davidson y Donald en Escocia (6) la serie de Stpetoe (20) y los 3.600 casos reportados durante 1968-1972 por Wheless y Thompson en Baltimore, USA. (23).

\section{Material y Resultados}

A finales de 1973 organizamos y llevamos a cabo dos seminarios de adiestramiento en procedimientos laparoscópicos. El uno, en el Instituto Materno Infantil de Bogotá, Hospital Universitario de gran volumen y el otro, en el Hospital de San Rafael en Girardot, un hospital de características regionales que sirve un área rural. En cada uno de estos seminarios se entrenaron a profesionales los cuales hasta el 30 de Junio de este año, 1974 habían practicado un total de 534 laparoscopias. El propósito de este artículo es presentar un informe preliminar sobre las primeras 100 laparocsopias practicadas en el Departamento de Ginecología y Obstetricia del Instituto Materno Infantil de Bo- 
Vol. XXV

No 3

ESTERILIZACION FEMENINA AMBBULATORIA POR LAPAROSCOPIA BAJO ANESTESIA 213

gotá, en forma ambulatoria, bajo anestesia local y sedación sistémica. En 6 de estos casos se hizo la Laparoscopia como procedimiento diagnóstico y en 94 se practicó para esterilización voluntaria por coagulación y sección de las trompas.

Todas las pacientes eran multíparas, de más de cuatro hijos vivos y por lo general con edades por encima de 30 años, que solicitaron voluntariamente la anticoncepción definitiva, autorizándola en carta firmada por ambos cónyuges.

\section{ESTUDIO SOBRE LAPAROSCOPIA \\ Instituto Materno Infantil (IMI) Bogotá}

\section{RESIDENCIA}

Urbano mismo Municipio
Rural mismo Municipio
Urbano otro Municipio
Rural otro Municipio
Fuera del país
TOTAL

EDAD AGRUPADA

\begin{tabular}{cccccc}
\hline $19-24$ & $\mathbf{2 5}-29$ & $\mathbf{3 0}-\mathbf{3 4}$ & $\mathbf{3 5}-\mathbf{3 9}$ & $\mathbf{4 0}-\mathbf{4 5}$ & Total \\
\hline $6^{*}$ & 14 & 33 & 32 & 15 & 100 \\
\hline
\end{tabular}

\section{ESTADO CIVIL}

\section{Nunca en unión}

Actualmente en unión

Estuvo en unión

TOTAL

$\begin{array}{r}76 \\ 1 \\ 15 \\ 7 \\ 1 \\ \hline 100\end{array}$

T.

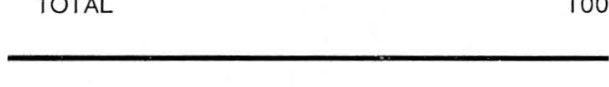

TOTAL NACIDOS VIVOS

\begin{tabular}{lccccc}
\hline 0 & 3 & $4-6$ & 7 & y & Total \\
\hline $6^{*}$ & 3 & 44 & 47 & 100 \\
\hline * Estos casos & con edades de 19 a 24 y con \\
$\begin{array}{l}\text { cero (O) hijos } \\
\text { diagnósticas. }\end{array}$ & &
\end{tabular}

Se presentan a continuación los cuadros que resumen algunas de las características y resultados de estos primeros 100 casos de Laparoscopia Ambulatoria.

\section{NUMERO DE ABORTOS}

\begin{tabular}{ccccc}
\hline $\mathbf{0}$ & $\mathbf{1 - 2}$ & $\mathbf{3 - 4}$ & $\mathbf{5} \boldsymbol{y}+$ & Total \\
\hline 45 & 36 & 14 & 5 & 100 \\
\hline
\end{tabular}

TECNICA USADA

\begin{tabular}{lr}
\hline Fulguración bilateral & 91 \\
Fulguración trompa izquierda & 3 \\
Laparoscopia diagnóstica & 6 \\
& \\
TOTAL & 100 \\
\hline
\end{tabular}

\section{DIFICULTADES QUIRURGICAS}

\begin{tabular}{lr}
\hline Ninguna & 98 \\
Plastrón anexial & 1 \\
Paciente obesa & 1 \\
\cline { 2 - 2 } TOTAL & 100 \\
\hline
\end{tabular}

COMPLICACIONES

(Procedimiento Laparoscópico)

\begin{tabular}{lr} 
Ninguna & 92 \\
Fiebre $+38^{\circ}$ & 3 \\
Hemorragia & 1 \\
Mareos & 2 \\
No dice cuál & 2 \\
$\quad$ TOTAL & 100 \\
\hline
\end{tabular}

OTRAS COMPLICACIONES

(Seguimiento)

\begin{tabular}{lr}
\hline Mareos & 1 \\
Ninguna & 1 \\
U. R. & 98 \\
TOTAL & 100 \\
\hline
\end{tabular}


RESUMEN DE LOS SEIS CASOS DE LAPAROSCOPIA DIAGNOSTICA

\begin{tabular}{|c|c|c|c|c|c|}
\hline Historia No & Edad & Sintomatología & Examen y Diag. $\mathrm{Cl}$. & Ginecografía & Laparoscopia \\
\hline H.N. 669500 & 31 & Amenorrea primaria & Disgenesia ovárica & $\begin{array}{l}\text { Utero y ovarios } \\
\text { pequeños }\end{array}$ & $\begin{array}{l}\text { No se visualizaron ovarios (Laparotomía } \\
\text { posterior mostró } 2 \text { pequeñas masas ová- } \\
\text { ricas) }\end{array}$ \\
\hline H.N. 526025 & 24 & $\begin{array}{l}\text { Oligomenorrea } \\
\text { Hirsutismo }\end{array}$ & Ovarios poliquísticos & Ovarios aumentados & $\begin{array}{l}\text { Ovario derecho aumentado } \\
\text { Quiste paraovárico izquierdo }\end{array}$ \\
\hline H.N. 668717 & 22 & Amenorrea primaria & Agenesia? & $\begin{array}{l}\text { No hay útero } \\
\text { imágenes laterales } \\
\text { de ovario }\end{array}$ & $\begin{array}{l}\text { No hay útero } \\
\text { Ovarios tamaño normal }\end{array}$ \\
\hline H.N. 285800 & 22 & Oligomenorrea & Ovarios poliquísticos & $\begin{array}{l}\text { Aumento ovario } \\
\text { derecho }\end{array}$ & Utero y ovarios normales \\
\hline H.N. 593110 & 20 & $\begin{array}{l}\text { Hirsutismo } \\
\text { Obesidad }\end{array}$ & $\begin{array}{l}\text { Ovarios poliquísticos? } \\
\text { S. Cushing? }\end{array}$ & Ovarios aumentados & $\begin{array}{l}\text { Ovarios tamaño normal } \\
\text { Superficie lisa }\end{array}$ \\
\hline H.N. 662795 & 18 & Oligomenorrea & Disgenesia ovárica & & Ovarios aspecto normal \\
\hline
\end{tabular}




\section{Técnica del procedimiento}

Con el fin de que pueda servir de guía 0 ayuda en adiestramientos futuros y como información general que pueda orientar a las nuevas pacientes en la comprensión del procedimiento utilizado, se describen a continuación en detalle los pasos que se han utilizado rutinariamente en la casuística presentada.

\section{Pre-Operatorio}

Se ha adoptado una hoja de historia clínica especial que consigna en forma pre-codificada los datos de la paciente. En general se hace hincapié en que el último parto haya tenido lugar 60 días antes por lo menos y se han descartado casos con trastornos cardíacos o pulmonares graves, cicatrices abdominales extensas, inflamación pélvica $u$ obesidades de más de 80 kilogramos.

Dos o tres días antes del procedimiento, se hace examen pélvico para descartar cualquier patología reciente $y$ se interroga sobre la fecha de la última menstruación y el uso de anticonceptivos, para tratar de asegurarse de manera más certera sobre la ausencia de un embarazo, que aunque no es contraindicación, pociría ser causa de (falsas) fallas atribuídas al procedimiento. En caso de duda se prefiere practicar prueba de progesterona previamente.

La noche antes de la admisión, la paciente deberá cenar en forma ligera y hacer un baño general con limpieza meticulosa" en la zona abdominal y en particular de la zona umbilical. Se aplica también un enema o un supositorio laxante.

\section{En la mañana de admisión:}

La mañana de admisión: la paciente se presenta en ayunas en la ofici- na de admisión, a las 7:30 a.m. La secretaria la lleva a una habitación cercana a la sala de Laparoscopia, situada dentro del hospital pero fuera del área quirúrgica, para que cambie sus ropas de calle y espere el turno de cirugía. No se rasura abdomen ni periné. Antes de pasar caminando a la sala de Laparoscopia evacúa la vejiga espontáneamente.

\section{La intervención:}

La paciente se coloca sobre la mesa quirúrgica en posición de litotomía dorsal, con las nalgas 10 a 20 centímetros fuera de la mesa, para poder manipular el útero en cualquier dirección con la cánula de insuflación. Se inicia luego perfusión intravenosa con dextrosa al $5 \%$ y por la misma vía se aplican lentamente 100 miligramos de Meperidina y 10 miligramos de Diazepan*. Se lava con agua y jabón la zona umbilical y periumbilical aplicando luego una solución de alcohol yodado o cualquier otro antiséptico. Simultáneamente con la ayuda de un espéculo vaginal, se pinza el labo anterior del cuello uterino con una pinza de Jacob's y se inserta una cánula de Rubins intrauterina para manipular los órganos pélvicos en caso de ser necesaria una mejor visualización de éstos. El espéculo se retira tan pronto se coloca la cánula.

\section{Anestesia local:}

Se coloca un campo estéril sobre el abdomen y se aplican 10 centímetros de Xilocaína al $2 \%$ sin epinefrina subcutánea intradérmicamente en el borde inferior del ombligo.

* Ultimamente se ha utilizado en vez de éstos, dos y medio centímetros intravenosos de "Thalamonal" (Fentanyl y Droperidol) de Janssen. 


\section{Incisión :}

Con dos pinzas de campo se sujeta la curvatura inferior del ombligo y con bisturí se hace una incisión de un centímetro o menos en piel y tejido celular subcutáneo.

\section{Neumoperitoneo:}

La introducción de un gas, el Co2 en la cavidad abdominal es esencial para la Laparoscopia. El neumoperitoneo resultante hace que la cavidad abdominal se distienda y en la posición de Trendelenburg, los intestinos caigan hacia el diafragma, permitiendo la mejor visualización y el acceso más fácil a los órganos pélvicos. El neumoperitoneo se logra con la inserción de una aguja de Touhy a través de la incisión previamente hecha, ya que en este punto se reduce el riesgo de puncionar vasos muy grandes o de herir el intestino. Para determinar si la aguja ha penetrado a la cavidad abdominal, se retira el mandril y se colocan 2 o 3 gotas de suero fisiológico $\circ$ agua destilada estériles, dentro de la aguja hasta que ésta se llene, se hace tracción hacia arriba con las pinzas de campo y si el suero desaparece de la aguja es porque ha penetrado a la cavidad abdominal. Se conecta luego la aguja al tubo de gas y se introducen dos y medio a tres y medio litros de $\mathrm{Co} 2$ como máximo a razón de un litro por minuto. Por razones de seguridad el Co2 proviene indirectamente de un taque especial de distribución.

\section{La Laparoscopia:}

Luego de haberse establecido el neumoperitoneo se coloca la paciente en posición de Trendelenburg, la cabeza más baja que el abdomen con una inclinación de 20 a 30 grados. Esto permite que los intestinos se aparten de la pelvis proporcionando así la visualización de las estructuras anexiales. Se retira la aguja del neumoperitoneo y se introduce por la misma incisión y perpendicularmente a la pared abdominal el trocar. Al retirar el mandril del trocar debe permitirse el escape de una pequeña cantidad del $\mathrm{Co} 2$ del neumoperitoneo, para confirmar la posición intraabdominal del instrumento. Se introduce luego el laparoscopia a través de la camisa del trocar y se une el cable fibro-óptico a la fuente de luz. Es preciso cerciorarse de nuevo, de si se está dentro de la cavidad abdominal, para lo cual al mirar al través del laparoscopio deben visualizarse estructuras intra-abdominales, como asas intestinales, epiplón, etc. Se introduce luego un poco más el laparoscopio para visualizar la pelvis. La punta del laparoscopio ha podido ser previamente calentada con solución salina o toallas húmedas calientes para prevenir el opacamiento de la lente al entrar en contacto con la mayor temperatura de la cavidad abdominal. Si la lente se empaña durante el procedimiento, se puede tocar el instrumento con el fondo del útero $u$ otra estructura intra-abdominal para liberarlo de condensación. Con la pinza de coagulación introducida dentro del laparoscopio y el cable conectado se está listo para el paso siguiente.

\section{Electro-coagulación de las Trompas:}

En principio se ha seguido la técnica del Hospital de John Hopkins en Baltimore, para la cual se agarra con la pinza el istmo de la trompa uterina (más o menos a 2 centímetros del cuerno) aislándola de la pelvis y de las asas intestinales y se electrocoagula pisando el pedal de coagulación por unos 10 segundos, hasta que se blanqueen unos 2 centí- 
metros de trompa. Con mayor tracción se llega a seccionar la trompa. Es necesario que el operador tenga la certeza de que la trompa ha sido dividida en su totalidad, viendo perfectamente tanto el cabo proximal como el cabo distal del oviducto separado. Se ejecuta luego el mismo procedimiento en la trompa contralateral.

Las maniobras con el tenáculum y la cánula de Rubins puesta previamente en la cavidad uterina, facilitan al llevarla hacia uno u otro lado la visibilidad de las trompas para poderlas agarrar con el fórceps. Una vez electro-coaguladas y seccionadas ambas trompas, se retira la pinza de clectro-coagulación hacia afuera y se revisa totalmente la pelvis. El retiro de la pinza antes que el laparoscopio ayuda a evitar accidentes de quemadura en asas o en otras estructuras pélvicas en el momento de la revisión. Si durante ésta se encuentra algúin punto sangrante en los muñones de las trompas puede electro-coagularse de nuevo con toda confianza. Se retira enseguida el laparoscopio sin la camisa, la cual es preciso dejar en su sitio para permitir el escape de la mayor cantidad posible de Co2 intra-abdominal, lo cual disminuye molestias que las pacientes puedan sentir posteriormente. Se retira luego la camisa y se coloca un punto subcutáneo de Catgut simple dos ceros en la incisión de la piel. Esta se cubre con una curita o pequeño apósito. Se retira luego el tenáculo de Jacob's y la cánula de Rubins del útero. El tiempo transcurrido en promedio durante el acto operatorio ha sido de io minutos.

\section{Cuidados post-operatorios:}

La paciente sale en camilla* para la pieza. Se controlarán los signos vitales, se hace deambulación precoz y se suministra dieta líquida. Cuatro a seis horas después de ejecutado el procedimiento y revisada la paciente, se le clá la salida del hospital.

\section{Cuidados en la casa:}

Generalmente ninguno. Administración de un analgésico cualquiera si se considera necesario.

\section{Controles:}

Se citan las pacientes alrededor de 28 días después, a los seis meses y al año. En aquelos casos en que el cirujano por dificultades en el procedimiento sospeche que alguna de las irompas no fue completamente seccicnada, es conveniente practicar histero-salpingografía para mayor seguridad. Sin embargo, este procedimiento no debe ser ejecutado antes de los tres meses de haber sido hecha la Laparoscopia, pues podría permeabilizar los muñones secionados de la trompa. Es obvio que durante estos tres meses la paciente debe usar algún método anticonceptivo.

\section{Discusión}

La mayor diferencia en la técnica operativa se refiere al grado y punto de acción sobre las trompas de Falopio. La mayoría de los cirujanos escogen el itsmo de la trompa uterina, pero algunos no están de acuerdo y así Fragemheim (18) menciona el riesgo de coagular la trompa en el itsmo porque allí se encuentra una ramificación de la arteria uterina que podría lesionarse y causar hemorragia. Antes de cortar el itsmo, Steptoe (20) coagula extensamente en ambas direcciones alejándose del sitio de la pinza, incluyendo el mesosalpinx, de suerte que los vasos sanguíneos loca-

* Con la neurolepsia por Thalamonal las pacientes salen caminando. 
lizados debajo de la trompa uterina son también coagulados $y$ hay menos peligro de hemorragia. A los 2 centímetros de distancia de donde la trompa se hace pálida, él hace la sección entre las zonas cauterizadas.

Chun (5) y colaboradores informan que ellos coagulan y cortan en la extremidad proximal de la trompa. Boer (3) y Dunster seccionan la trompa en la extremidad lateral. Donald (7) secciona las trompas de Falopio cerca del cuello uterino sin dañar el suministro de sangre al mesosalpinx, él coagula con diatermina los muñones de la trompa pero conserva la longitud de las trompas de Falopio lateralmente y la circulación sanguínea se mantiene intacta con el fin de tener suficiente longitud de trompa sana para lograr reanastomosis posteriores si se desea.

Aunque la esterilización femenina puede ser en teoría reversible, en realidad la mayoría de los médicos consideramos la intervención irreversible y no se espera fecundidad posterior.

Existen diferencias así mismo entre las corrientes eléctricas aplicadas a los oviductos. Cohen (4) y colaboradores aplican una corriente moderada cortante una vez que la trompa es cogida con la pinza de biopsia. Wheeless (22) aplica una corriente cortante durante 10 segundos, porque observó que no era necesario que la trompa se necrose y caiga. La energía eléctrica cortante generalmente se considera más segura que la coagulante por ser más bajo el voltaje necesario, pero tiene la desventaja de que deja un muñón en la trompa que puede causar hemorragias posteriores. Golditch (9) utiliza inicialmente energía coagulante y luego una corriente cortante, de suerte que el segmento extirpado queda menos quema- do y claramente se puede identificar como trompa de Falopio.

Fragemheim (8) recomienda una corriente de coagulación baja, porque la corriente de alta frecuencia puede coagular el oviducto de manera incompleta dejando una parte intacta y permeable.

Wheeless (23) y colaboradores hacen notar que la proporción de fracasos cuando se utiliza sólo el cauterio es inversamente proporcional al tamaño de la sección de trompa cauterizada. Sin embargo, si se cauteriza demasiado, se aumenta el riesgo de complicaciones.

Otros de los puntos en los cuales hay diversidad de opiniones es acerca de si se debe coagular, coagular y seccionar o seccionar y extirpar una parte de las trompas de Falopio.

Quienes son partidarios del uso exclusivo de la electrocoagulación de los oviductos, insisten en que con ella ocurren menos hemorragias, hay menos trauma y es menor el peligro de producción de fístulas tubáricas. Opinan que si la electrocoagulación es completa y extensa, no puede ocurrir la recanalización de las trompas. Jorclán (10) y colaboradores ensayaron la electrocoagulacióni sola y la electrocoagulación adicionada de sección de las trompas en 1, 2 y 3 puntos de longitud y llegaron a la conclusión, al final de su estudio con 910 pacientes, que la sección de las trompas al hacer la esterilización no es necesaria ni deseable. Sin embargo, hoy en día los partidarios de esta última técnica son muy escasos.

Muchos de los médicos que coagulan y seccionan las trompas lo hacen porque han observado permeabilidad tubárica en algunas pacientes después de la operación en la cual únicamente se electrocoaguló. Así, en 
los estudios de Peterson y Beherman (16) quienes trataron a 4 pacientes con electrocoagulación, una de ellas concibió tres meses más tarde, por lo cual en todos sus casos posteriores estos autores seccionaron las trompas.

Steptoe (21) encontró en la mitad de 50 casos tratados, pacientes con oviductos abiertos aún después de haber sido tratados con electrocoagulación exclusivamente. Liston y colaboradores (11) utilizaron la electrocoagulación sóla en 30 pacientes durante un período de 3 meses, antes de 1966, pero cambiaron al sistema de electrocoagulación con sección de los oviductos a causa de la permeabilidad tubárica persistente en un 10\% de sus pacientes.

En general, la mayoría de los autores concluyen que no hay problemas de hemorragias serias en la sección tubárica después de coagular si la operación es debidamente practicada. Algunos de los operadores hacen la operación coagulando, seccionando y extirpando una parte de las trompas con el fin de obtener una porción para examen histológico.

\section{Resumen}

Se menciona la organización de dos seminarios de adiestramiento en Laparoscopia y el establecimiento de ella en dos centros hospitalarios, uno en un Hospital Universitario, de gran volumen y otro, en un Hospital de características regionales. Se presentan los primeros cien casos de Laparoscopia y esterilización tubárica en forma ambulatoria con anestesia local en el Instituto Materno Infantil de Bogotá. Se describe en detalle la técnica usada y se dan algunos resultados preliminares.

En esta primera comunicación se han querido recalcar varios puntos:
1. La importancia de poder hacer Laparoscopia en pacientes ambulatorias, con mínimos costos tanto para la paciente como para la Institución.

2. El hecho de poder practicar el procedimiento bajo anestesia local y analgesia intravenosa concomitante con mínimos costos de derechos de sala de cirugía o de anestesia general.

3. La pequeñísima incisión infraumbilical que prácticamente no deja cicatriz.

4. La casi ninguna complicación del procedimeinto SI SE HACE CON EL MAXIMO CUIDADO Y CON LA EXPERIENCIA DEBIDA.

No debe terminar este trabajo sin agregar antes con Wheeless y Thompson (22) que la esterilización tubárica por Laparoscopia no es la respuesta final para el control permanente de la fecundidad. Sin embargo es un procedimiento efectivo no muy caro, de relativa fácil técnica, que puede ser efectuado con un alto porcentaje de seguridad cuando se hace con la debida técnica, experiencia, instrumentación y control apropiado.

Claro está que la serie comentada de casos es muy pequeña pero únicamente se ha querido presentar un nuevo servicio y una pequeña guía para todos aquellos futuros laparoscopistas. La intención es continuar informando periódicamente sobre los resultados y el manejo en nuevas técnicas, indicaciones, contraindicaciones y fallas del procedimiento.

\section{BIBLIOGRAFIA}

1 ANDERSON, E. T., "Peritoneoscopy". American Journal of Surgery. 35: 36-39, 1937.

2 BATHIA, D., "Role of Sterilization Operations in Family Planning". Pakistan Internatlion Family Planning Conference, En. 28 Reb. 4, 1969, Dacca. 
3 BOER, C. H. and DUNSTER, G. "Tubal Electrocoagulatin and Assessment". American Journal of Obstetrics and Gynecology, 3 (7) 979-983, Diciembre 1\%, 1971.

4 COHEN, MELVIN, R., TAYLOR, MARSHALL BERMENT, and KASS, MARTIN, B. "Internal Tubal Sterilization via Laparoscopy". American Journal of Obstetrics and Gynecology. 109 (5) 794-809, Marzo 19, 1971.

5 CHUN, DAPHNE, M. A., H. K., VIP, J. K., and CHAN, P. K. "Laparoscopic Tubal Sterilization". International Planned Parenthood Federation (I.P.P.E.) Medical Bulletin 4: 1-3, Junio de 1970.

6 DAVIDSON, A. C. and DONALD, I. "Female Sterilization". Scottish Medical Journal, 17 (6) 210-213, Junio 1972.

7 DONALD, IAN, GREEN, G. D., and BAIN, W. H. "Female Sterilization". British Medical Journal 4. 170. Octubre 19 de 1968.

8 FRAGEMHEIM, H. "Tubal Division Under Endoscopic Control". German Medical Monthly. 9: 421-422, 1964. English translation of: Die Tubensterilization under Sicht mit dem Lalaroskop, Geburtschilfe und Fravenhei. 24: 270-471, 1964.

9 GOLDITCH, IRA, M. "Laparoscopy: Advances and Advantages, Fertility and Sterility". 22 (5): 306-310, May. 1971.

10 JORDAN, J. A., EDWARD, R., LOGAN PEARSON, J., and MASKWERY P. J. K. "Laparoscopic Sterilization and Follow-Up Histerosalpingogram". The Journal of Obstetrics and Gynecology of the British Commonhealth. 78: 440-466, Mayo 1971.

11 LISTON, W. A., BARDFORD, W., DOWNIE, J. and KERR $M$.G. "Female Sterilization by Tubal Electro-Coagulation Under Laparosccpic Control". The Lancet. 382-383. Feb. 21 1970.

12 NOONAN, J. T., Contraception, Harvard Press Ed. Pág. 11, Cambridge, Mass. 1966.
$13 \mathrm{PAl}$, D. N., "Volnntary Sterilization as a Component of Family Planning". Key Note Address 2. Inf. Conform Voluntary Sterilization. Feb. 25 - Marzo 19 1973. Ginebra.

14 PALMER, R. "Instrumentation et technique de la Celiescopie Gynecologique". Gynecology Obst. (Paris) 46: 420-431, 1947.

15 PALMER, R. "Les Techniques Chirurgicale et Coelioscopiques de Sterilisation Tubaire et Leurs Résultats". Association National pour L'Etude de L'Avortement, Paris, International Conference of Contraception, Steri ${ }^{-}$ lization and Abortion. Mayo 1972.

16 PETERSON, E. P. and BEHRMAN, S. J. "La, paroscopic Tubal Sterilization". American Journal of Obstetrics and Gynecology. 110 (1): 24-31, Mayo 1\%, 1971

17 POWER and BARNES, A. C., "Sterilization by Means of Peritoneoscopic Tubal Fulguration". American Journal of Obstetrics and Gynecology. 41: 1038-43, 1941.

18 STEPTOE, P. C., Laparoscopy in Gynecology. London: E.T.S. Livingston, Ld. 1967, pp. 150 .

19 STEPTOE, P. C. "Laparoscopic Sterilization in Cursen Peter: Report of Scientific Meeting. Royal College of Obstetrics. Journal of Obstetrics and Gynecology of the British Commonwealth 76: 1043, Noviembre, 1969.

20 STEPTOE, P. C. "Laparoscopic Tubal Sterilization, a British Viewpoint, I.P.P.F. Medical Bulletin 5: 4 Abril, 1971.

21 STEPTOE, P. C. "Problems of Laparoscopic Sterilization". The Lancet. 1: 1115, Mayo 20, 1972.

22 WHEELESS, C., CLIFFORD, R., Jr. Outpationet Laparoscope Sterilization Under Local Anesthesia, Obstetrics and Gynecology. 39 (5): 767-770, Mayo 1972.

23 WHEELESS C., and THOMPSON B. "Laparoscopic Sterilization". Review of 3.600 Cases, Obstet. and Gynecology. 42: 751-758, 1973. 